\title{
Review
}

Pernille J. Vinholt*

\section{The role of platelets in bleeding in patients with thrombocytopenia and hematological disease}

https://doi.org/10.1515/cclm-2019-0380

Received April 8, 2019; accepted July 29, 2019; previously published online August 29, 2019

Abstract: This review evaluates the role of platelets in bleeding risk among patients with hematological disease and thrombocytopenia. Platelets are pivotal in primary hemostasis, and possess non-hemostatic properties involved in angiogenesis, tissue repair, inflammation and metastatis. Also, platelets safeguard vascular integrity in inflamed vessels. Overall, bleeding risk depends on the underlying disease, and patients with cancer and platelet count $<6-10 \times 10^{9} / \mathrm{L}$ have a markedly increased bleeding risk, while the platelet count does not correlate with bleeding risk at higher platelet counts. Other factors might affect platelet properties and thus bleeding risk, for example, drugs, low hematocrit, coagulation system impairments or transfusion of dysfunctional donor platelets. For patients with leukemia and immune thrombocytopenia, reduced platelet activation, platelet aggregation, or thrombopoiesis, reflected by the reduced presence of reticulated platelets, are associated with bleeding phenotype. However, mechanistic insight into the cause of reduced platelet function in different thrombocytopenic conditions is sparse, except for some inherited platelet disorders. Promising tools for platelet function studies in thrombocytopenia are flow cytometry and biomarker studies on platelet constituents. An important message from this current paper is that bleeding risk assessment must be tailored to specific patient populations and cannot be applied broadly to all patients with thrombocytopenia.

Keywords: hemorrhage; neoplasms; platelet count; platelet function tests; thrombocytopenia.

\footnotetext{
*Corresponding author: Pernille J. Vinholt, MD, PhD, Associate Professor, Department of Clinical Biochemistry and Pharmacology, Odense University Hospital, J.B. Winsløws vej 4B, 5000 Odense C, Denmark, Phone: +4529648694, E-mail: pernille.vinholt@rsyd.dk. https://orcid.org/0000-0002-2035-0169; and University of Southern Denmark, Odense, Denmark
}

\section{Introduction}

Platelets are pivotal in hemostasis [1]. Accordingly, patients with hematological disease and thrombocytopenia experience increased bleeding risk. To avoid bleeding, much effort has been put into prophylactic strategies, focusing mainly on the platelet count as a trigger for transfusion among patients with hematological cancers and thrombocytopenia [2]. However, the platelet count is not the only determinant of bleeding risk [3-5]. Thus, other factors contribute to the bleeding risk. In this regard, the platelet function might be relevant, as impaired platelet function would reduce the hemostatic capacity of platelets. The current literature suggests that impaired platelet function could be relevant for bleeding in thrombocytopenia; a relationship which has been particularly explored in hematological disease [6-10]. When interpreting the existing literature, it is, however, important to consider which methods have been used for the evaluation of platelet properties and platelet function, as some methods do not produce reliable results in samples with low platelet count.

The aim of the current review is to evaluate the role of platelet function in bleeding risk among patients with hematological disease.

\section{Bleeding in hematological disease}

In 1962, Gaydos et al. studied 92 patients with acute leukemia, and found an increasing bleeding risk with declining platelet count [11]. Others reported that bleeding was a contributing cause of death in $67 \%$ of cases with leukemia [12]. At that time, platelet transfusions were casuistically shown to treat bleeding in thrombocytopenia, as first reported by Duke in 1910 [13]. Platelet transfusions became widely available during the 1960 s and 1970 s and the platelet count became the main transfusion trigger.

While bleeding is frequent in hematological cancers with bone marrow failure [5], bleeding is thought to be rarer in thrombocytopenic conditions with high platelet turnover. Thus, bleeding was found in $14 \%, 6 \%$, and $12 \%$ of admissions for thrombocytopenic purpura (TTP), 
heparin-induced thrombocytopenia (HIT) and immune thrombocytopenia (ITP), respectively [14]. In these conditions, the platelet count drops when platelets are consumed through accelerated coagulation processes; and platelet transfusions increase the thrombosis risk and the mortality [14].

It shall, however, be mentioned that the definition of bleeding differs in published studies, which could influence the reported incidences of bleeding in thrombocytopenia [15]. Also, bleeding is notoriously challenging to document correctly as some mucosal bleeds remain occult; further, there is a risk of bias because the patients' recollection and retelling of the episodes are subjective. Hence, standardization in methods for registration of bleeding is much needed [15].

\section{The platelet count}

The platelet count is used for monitoring thrombopoiesis; it exhibits large inter-individual variation, but is relatively stable in healthy individuals with an overall slight decline with age $[16,17]$. Thrombocytopenia has been inconsistently defined in the literature as platelet count $<100 \times 10^{9} / \mathrm{L}$ or $<150 \times 10^{\%} / \mathrm{L}[18,19]$, and does not reflect the natural occurring age-, sex-, and ethnicity-dependent differences with a lower reference limit for platelet count around $125 \times 10^{9} / \mathrm{L}$ for men and $150 \times 10^{9} / \mathrm{L}$ for women with ethnical differences [17, 20]. Nevertheless, a consensus panel defined primary ITP as a platelet count $<100 \times 10^{9} / \mathrm{L}$ in the absence of other causes or disorders that may be associated with thrombocytopenia [19]. Thereby a limit has been set for what should be regarded as a pathologically low platelet count and consequently should result in diagnostic considerations.

A reduced platelet count has in numerous studies been confirmed to be a bleeding risk factor $[3,4,11,21]$. Spontaneous fecal blood loss was observed at platelet count $<10 \times 10^{9} / \mathrm{L}$, and increased markedly when the platelet count was $<5 \times 10^{9} / \mathrm{L}$ in a study among patients with aplastic anemia [22].

The platelet count is, however, not the only determinant for bleeding risk in thrombocytopenia. Two large studies in patients with hemato-oncological cancers congruently found that bleeding risk is increased at platelet counts $<80 \times 10^{9} / \mathrm{L}$ with no clear pattern of decreasing risk with increasing platelet counts [3, 4]. Even severe thrombocytopenia does not per se equal bleeding, as major bleeding was observed in only $31 \%$ of days with a platelet count $<1 \times 10^{9} / \mathrm{L}$ in patients with acute leukemia [11]. Hence, the current praxis of giving prophylactic transfusions at a platelet count $<10 \times 10^{9} / \mathrm{L}$ results in patient over-treatment. On the other hand, a no-prophylactic platelet transfusion strategy increases the overall risk of major bleeding [23, 24], but might be safe in patients undergoing autologous transplantations [23]. Thus, factors other than the platelet count contribute to bleeding risk in hematological disease.

\section{Mechanistic insight into properties of platelets to prevent bleeding}

\section{Platelet plug formation and bleeding - classic hemostatic properties}

Platelets are important in primary hemostasis as they adhere, spread, and aggregate at the site of vessel injury (Figure 1), which in the best case stops the bleed. In an injured vessel, platelets adhere to immobilized von Willebrand factor via platelet glycoprotein (GP)Iba $[25,26]$ and via collagen binding to $\alpha 2 \beta 1$ and GPVI [27]. During this process, platelets become activated and release platelet granules, change shape, and activate the fibrinogen receptor GPIIb/IIIa [1]. Granule content, particularly ADP, rapidly activates a large numbers of platelets [1]. The shape change results in platelet spreading, which limits bleeding and provides a surface for platelet aggregation [25]. When platelets aggregate, they are linked through binding of ligands from plasma to the GPIIb/IIIa receptor. Fibrinogen is the primary ligand for the GPIIb/IIIa receptor, but several other ligands contribute to platelet aggregate formation particularly at high shear, including von Willebrand factor and fibronectin [28]. The major platelet activation pathways are via binding of collagen to the integrin $\alpha 2 \beta 1$ and GPVI, thrombin via the protease-activated receptor (PAR)-1 and PAR-4 or ADP via the P2Y1 and P2Y12 receptors [25]. Vessel injury also engages the secondary hemostasis which leads to fibrin mesh formation that strengthens the clot [1, 25]. Via surface exposure of negatively charged phospholipid phosphatidylserine, platelets serve as an adhesion site for coagulation factors and enhance the efficiency of the coagulation reaction $[1,25]$.

\section{Platelets and the vasculature - extended hemostatic properties}

Evidence is mounting for several other platelet properties to be involved in angiogenesis, tissue repair, inflammation, and metastatis of cancer. However, these so-called 


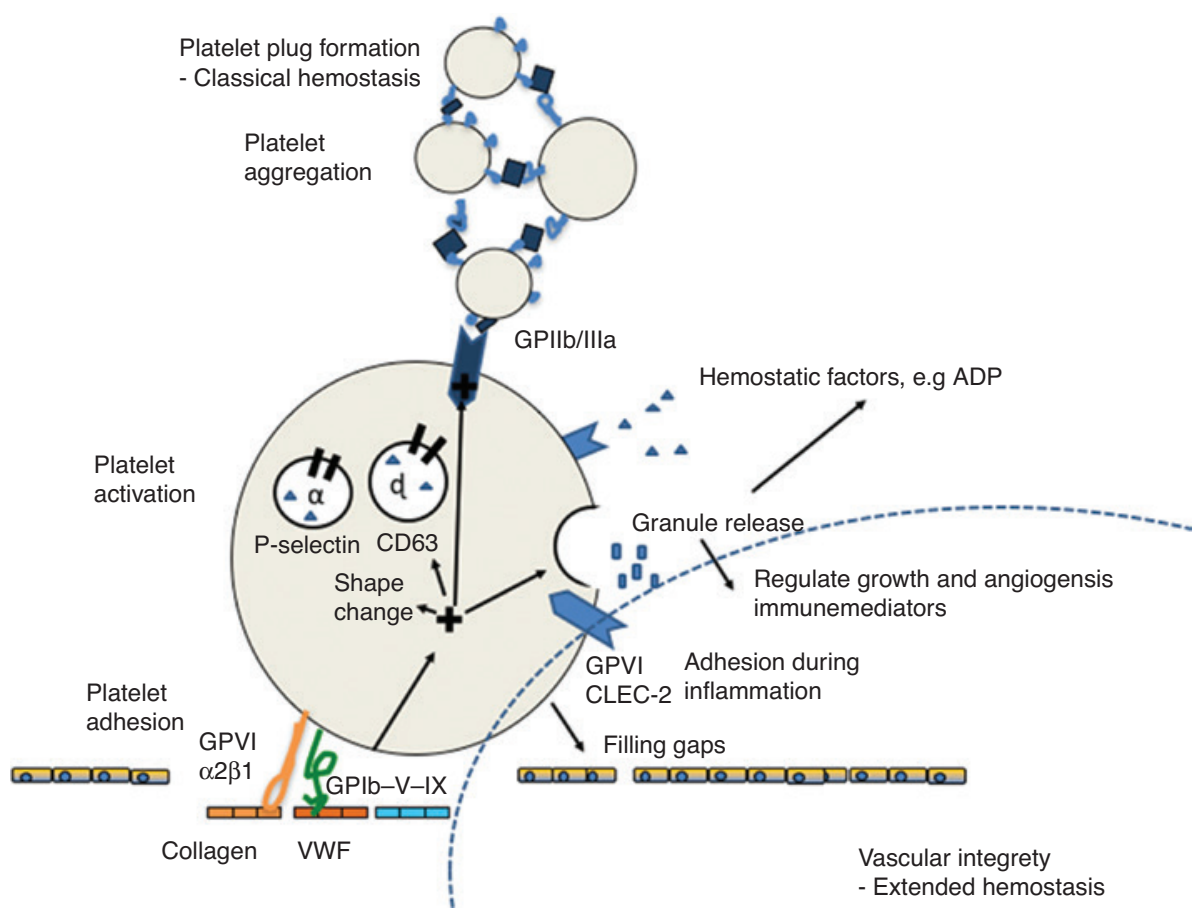

Figure 1: The platelet properties that prevent bleeding.

Platelets prevent bleeding via an effect on the vasculature and form plug to stop bleeding in case of injury (1) platelet plug formation - classic hemostasis: in an injured vessel, platelets adhere to immobilized von Willebrand factor via platelet glycoprotein (GP)Iba and via collagen binding to $\alpha 2 \beta 1$ and GPVI. Platelets are activated and release their granules, change shape and activate the fibrinogen receptor GPIIb/IIIa. Granule content rapidly activates large numbers of platelets. The shape change results in platelets spreading, which not only limits bleeding but also provides a surface for platelet aggregation. When platelets aggregate, they are linked through binding of ligands from plasma to the GPIIb/IIla receptor; (2) vascular integrity - extended hemostasis: platelets close gaps in the endothelium. Platelets secrete granules that contain proteins that regulate angiogenesis, wound healing and remodeling, immune cell recruitment and inflammation. These factors may regulate the vessel stability. The receptors glycoprotein VI and C-type lectin receptor 2 (CLEC-2) are critical in inflammatory hemostasis.

'non-hemostatic' properties appear to be relevant for the prevention of bleeding and a more fitting terminology would therefore be non-classic hemostatic properties or extended hemostatic properties. Circulating platelets support the barrier function of the resting endothelium by mechanically filling the gaps in the endothelial lining [29] (Figure 1). This is in accordance with the clinical observation that spontaneous blood loss in stool increases considerably with platelet counts $<5-10 \times 10^{9} / \mathrm{L}$ in patients with aplastic anemia [22].

In mice, thrombocytopenic bleeding typically occurs in areas with pre-existing inflammation which points to the role of platelets in vascular integrity during inflammation $[30,31]$. In a study with transgenic mice, the receptors glycoprotein VI and C-type lectin receptor 2 (CLEC-2) were critical in hemostasis in the inflamed skin and lung [32]. GPVI receptors have only a minor impact on hemostasis under normal circumstances in humans, as the lack of GPVI results in only a mild bleeding phenotype [33]. Further, a lack of platelet granules induced intracerebral bleeding in mice with ischemic brain infarction, but did not result in bleeding in experimental inflamed skin or lung models [31]. Thus, the platelet involvement in inflammatory hemostasis appears to be tissue-specific.

Interestingly, platelet granule secretion also prevented thrombocytopenia-induced tumor bleeding [34]. This is related to the content of platelet granules, including regulators of tissue repair, immune responses, and vasoconstriction that are differentially and selectively secreted in an active manner $[1,35]$. Platelets constituents either originate from the megakaryocytes, are synthesized de novo, or are taken up from the platelets' surroundings. Albeit platelets are anucleate, they express RNAs and are able to translate mRNAs into proteins. Further, they are capable of intercellular transferring of microparticles and RNAs, which might regulate cellular functions [36]. Moreover, the processes by which platelets maintain vascular integrity at the site of inflammation appears to be independent from the ability to form clots [30, 32]. These important observations permit studies of individualized treatment based on the clinical disease spectrum. 


\section{Why do patients with hematological disease have impaired platelet function?}

For some inherited platelet disorders there is a welldescribed association between the molecular defects and the phenotype. In inherited platelet disorders, specific genetic mutations result in changes in platelet composition; some of which are associated with increased bleeding risk [37]. For example, absence of platelet $\alpha$-granules is observed in cases with mutations in NBEAL2 and is characterized by a mild to moderate bleeding phenotype [38]. Other categories of patients with thrombocytopenia might experience impaired platelet function, which has primarily been investigated among patients with acute myeloid leukemia (AML) and ITP, but mechanistic insights into the nature of these acquired platelet function defects are sparser. A brief overview of platelet function in hematological disease is shown in Table 1.

Whether platelet function defects are mainly introduced by mechanisms related to the underlying disease, remains to be established. However, other blood constituents such as drugs, coagulation defects, changes in red blood cells, and platelet transfusions might also impair platelet function in thrombocytopenia. For example, up to $45 \%$ of admitted patients with thrombocytopenia receive antiplatelet or anticoagulant therapy [46].

Further, other factors influence the overall hemostatic capacity of platelets and contribute to bleeding risk. Impairments in the coagulation system could decrease the thrombin generation potential and thus reduce the stimulus for platelet activation in vivo, while an induction of fibrinolysis would reduce clot strength [47]. It may be speculated that a compensatory rise in von Willebrand factor to some extent could compensate for the platelet functional defect. Red blood cells influence platelet aggregation capacity in vitro [48]. While studies are lacking, it is important to keep in mind that the matrix for platelets influences the test result when choosing a platelet function test.

The rheological properties of blood are important for limiting bleeding in vivo, but is not evaluated in any available method. To execute their hemostatic function, platelets must be placed close to the vessel wall. During flow, red blood cells cause marginalization of platelets and thus increase the platelet concentration at the site of action. Accordingly, low hematocrit is a risk factor for bleeding in thrombocytopenia [4, 46, 49].

Thrombocytopenic patients might receive platelet transfusion and it is evident that in vitro platelet function declines during storage of platelet concentrates for transfusion [50]. Thus, it is a concern whether variations in platelet function of platelet concentrates affect bleeding risk in recipients with thrombocytopenia. Unfortunately, no model accurately predicts the interplay between donor and patient platelets or the hemostatic effects of transfused platelets [50, 51]. A recent study found that transfused platelets are not cleared from the bloodstream dependent on the platelet function in vitro [52]. While this area remains to be fully studied due to methodological limitations of platelet aggregation tests during thrombocytopenia, it would be very relevant for the interpretation of recent studies of bleeding in thrombocytopenia.

Table 1: Platelet function in hematological disease.

\begin{tabular}{ll}
\hline Disease & Platelet function \\
\hline Acute myeloid & - Reduced platelet count and IPF \\
leukemia & - Reduced platelet activation determined as the percentage of platelets positive for or the expression of activated \\
& GPIIb/IIIa, CD63, and P-selectin before and after stimulation with TRAP, collagen-related peptide and ADP [6, \\
& $39,40]$ \\
& - Reduced GPIb expression [6, 39, 40] \\
& - Reduced platelet aggregation with ADP, TRAP and collagen-related peptide, associated with bleeding [6, 10] \\
& - Low platelet count and high IPF, reduced adhesion and clot strength with viscoelastic methods [41-43] \\
Immune & Reduced platelet activation as determined either as the percentage of positive platelets or the expression of \\
thrombocytopenia & platelet activated GPIIb/IIla and P-selectin was found with TRAP, and convulxin while data on ADP conflicts [7, 8, 44] \\
(ITP) & - Higher GPIb after stimulation with TRAP and ADP than normal [44] and high GPIb is associated with bleeding [8] \\
& - Reduced flow cytometric platelet aggregation with phorbol myristate acetate (PMA) in patients with severe \\
& bleeding symptoms [7] \\
- & Lack of specific platelet receptors or properties is diagnostic for various inherited platelet disorders, e.g. lack of \\
Others & functional GPIlb/IIla is seen in Glanzmann's thrombosthenia [37, 45] \\
hematological & - Specific knowledge is lacking in other hematological diseases \\
diseases &
\end{tabular}

ADP, adenosine diphosphate; GP, glycoprotein; IPF, immature platelet fraction; TRAP, thrombin-receptor activating peptide. 


\section{Current methodology and clinical studies of platelet function in patients with thrombocytopenia and hematological disease}

\section{Platelet indices}

Platelet size, such as the mean platelet volume (MPV), might reflect functional platelet properties as large platelets exhibit higher platelet aggregation capacity in vitro than small platelets [53]. One study from 1982 proposed that low MPV identified bleeding in patients with hematological disease [54]. However, platelet size measurements have methodological limitations which restrict their use $[9,55]$ (Table 2) and newer studies were unable to show that MPV is a bleeding risk marker [7-9].

Immature platelets might be more hemostatically active than older platelets [69]. As immature, newly released platelets are rich in mRNA, they can be identified with fluorescence labeling [56]. Immature platelet fraction

Table 2: Methods for measuring platelet characteristics and function with focus on technical and clinical aspects.

\begin{tabular}{ll}
\hline Method & Technical aspects \\
\hline Platelet count $[3,56]$ & Available on automated hematological equipment \\
& $\begin{array}{l}\text { Available on automated hematological equipment. Size } \\
\text { enlarges during sample storage, lack of standardization, } \\
\text { Platelet size, e.g. MPV }\end{array}$ \\
$\begin{array}{l}\text { cannot be obtained in up to } 64 \% \text { of samples, inaccurate if } \\
\text { platelets are extraordinarily small or large } \\
\text { IPF }[41,56,57]\end{array}$ & Limited availability and lack of standardization
\end{tabular}

Clinical aspects

Low platelet count is associated with bleeding. However, the platelet count does not correlate with bleeding risk Not a suitable clinical marker due to technical limitations

Reflect thrombopoiesis and aids determining the cause of thrombocytopenia. Reduced IPF associated with bleeding as small platelets are less hemostatically active A potential source of novel biomarkers, but studies are lacking. Platelet secretion assays can aid diagnosing inherited platelet disorders

Reduced adhesion associated with bleeding risk as clot formation at site of vascular damage is reduced In vivo, reduced platelet aggregation is associated with a lack of clot formation and bleeding. A lack of studies on Multiplate and bleeding at low platelet counts. Large CV\% makes Multiplate less likely to be useful for decision making. LTA is not recommended

Lack of receptors might indicate functional problems and can be diagnostic for inherited platelet disorders. Reduced platelet activation and aggregation capacity is associated with bleeding

Not recommended for testing platelet function in thrombocytopenia

Low clot strength associated with bleeding. The independent value for bleeding risk assessment unknown as it might reflect low platelet count

CV, coefficient of variation; IPF, immature platelet fraction; LTA, light transmission aggregometry; MPV, mean platelet volume. 
(IPF, the percentage of immature platelets) is dependent on the cause of thrombocytopenia. A high IPF suggests increased thrombopoiesis, which is seen in conditions with peripheral platelet consumption or destruction, such as ITP or TTP [57]. In bone marrow failure, IPF is low and a rise may predict platelet recovery, for example, following hematopoietic progenitor cell transplantation [56]. Low IPF was associated with bleeding phenotype in ITP $[8,41,57]$; but only when platelet count was low. A recent study found that the absolute immature platelet number was lower in ITP patients with episodes of severe bleeding than in those without, despite similar platelet counts [57]. In patients with hematological cancer and a platelet count $\leq 50 \times 10^{\%} / \mathrm{L}$, a low absolute immature platelet number was associated with bleeding within the next day [9], while IPF did not identify bleeding risk in patients with cancer and thrombocytopenia in another study [46].

\section{Global tests}

The attempt of using global whole blood tests to mimic the in vivo hemostatic process is theoretically advantageous. In global assays, platelets are more or less indirectly activated dependent on the interplay of multiple hemostatic processes. Viscoelastic methods are global approaches which have gained ground in the monitoring of acute critical bleeding. It facilitates the timely and appropriate use of transfusions during acute critical bleeding [67]. Among other parameters, the clot strength is measured, a parameter that depends on the platelet count [67]. Low clot strength using viscoelastic methods identified the bleeding phenotype in severe thrombocytopenia of various causes [68] and in patients with ITP [41, 42]. Although these methods might be less sensitive for platelet function defects, Estcourt et al. found that results from viscoelastic methods predicted bleeding among patients with hematological cancer and thrombocytopenia, after adjustment for the platelet count [9]. Other currently available global tests are not useful for thrombocytopenic samples. The Platelet function Analyzer-100 ${ }^{\circledR}$ (PFA-100 ${ }^{\circledast}$, Siemens Healthcare Diagnostics GmBH, Marburg, Germany) is not applicable to samples with a platelet count $<50 \times 10^{9} / \mathrm{L}$ as the low platelet count prolongs the blood clotting time, which often rises above the upper measurement range [66]. Bleeding time ad modum Ivy is no longer recommended [60].

\section{Platelet adhesion}

Platelet adhesion is pivotal for both platelet plug formation and relevant for the extended hemostatic effects of platelets. Reduced platelet adhesion was associated with bleeding tendency in patients with thrombocytopenia [43, 59] and correlated with the severity of bleeding symptoms in ITP [59]. These studies used the Cone-and-Plate[let] analyzer (Impact- $\mathrm{R}^{\mathrm{TM}}$, DANED SA, Belgium) which is a commercially available platelet adhesion test. More clinical studies into platelet adhesion during thrombocytopenia would be relevant for insights into the disease mechanisms and could potentially reveal markers of bleeding as platelet adhesion is a critical process in hemostasis. However, the field is limited by the lack of commercially available easy operable tests that specifically reflect platelet adhesion.

\section{Platelet aggregation}

Platelet aggregation testing with light transmission aggregometry (LTA) remains the gold standard for platelet function testing and has been widely used $[60,61]$. It is, however, important to be aware that the method requires a platelet count on at least $150 \times 10^{9} / \mathrm{L}$ [61]. Nevertheless, LTA and PFA-100 ${ }^{\circledR}$ identified bleeding phenotype in persons with ITP and normal platelet counts [70]. Lumiaggregometry, an extension of the classical light transmission aggregometer, might be more sensitive to reduced platelet function at low platelet counts than LTA [71], but studies of bleeding risk in thrombocytopenia are lacking. The Multiplate ${ }^{\circledR}$ Analyzer (Roche Diagnostics $\mathrm{GmbH}$, Mannheim, Germany) is an impedance aggregometer that evaluates platelet aggregation in whole blood. Technically, the method can be used with samples with severely reduced platelet counts [62, 72], but its results are significantly reduced at platelet counts $<150 \times 10^{9} / \mathrm{L}$ [72], which, however, does not exclude a relationship with the bleeding phenotype. Studies among hematological diseases are lacking.

\section{Flow cytometry}

Importantly, the evidence is mounting for the use of flow cytometry to study platelet function. By flow cytometry each cell is characterized separately, and flow cytometry is thus applicable regardless the patient's platelet count [63, $65,73]$. The method has been widely used to study expression levels of surface receptors and to provide information about the platelet activation level in unstimulated and stimulated samples [65, 73, 74]. The platelet activation marker $\mathrm{P}$-selectin is an alpha-granule-membrane integrated receptor that is incorporated into the cell surface upon platelet activation [65, 73]. Platelet surface granulophysin (CD63) 
expression represents dense granule release and the expression of the activated GPIIb/IIIa receptor or fibrinogen binding to the receptor is a measure of the capacity for platelet aggregation. Platelet reactivity or activation capacity is reported as the change in expression of these platelet activation markers when platelets are stimulated in vitro with agonists [65]. Evaluation of Annexin $V$ has been used as a tool to study apoptosis [75]. Recently, it was further proposed that also platelet adhesion [64] and platelet aggregation, independent of the platelet count, could be measured with flow cytometry $[63,76]$.

Flow cytometry has been established as a part of the investigation for inherited platelet disorders, some of which are associated with bleeding risk [37]. Patients with ITP and a positive bleeding phenotype had higher levels of spontaneous platelet activation (platelet activation in unstimulated samples) [8], reduced platelet reactivity in terms of lower P-selectin expression [7, 8, 77], and low activated GPIIb/IIIa expression after platelet stimulation than ITP patients without bleeding $[8,77]$. Platelet function in ITP was consistent over time and was associated with both concurrent and subsequent bleeding severity [78]. Congruently, ITP patients with bleeding phenotype were shown to have decreased flow cytometric platelet aggregation [7]. However, a recent study showed that eltrombopag, a drug used for raising the platelet count, only had a minor impact on platelet function in ITP [44]. Overall, platelet activation capacity is higher in thrombocytopenic patients with ITP than AML [77].

For thrombocytopenic patients with AML or myelodysplastic syndrome, platelet activation capacity was lower than in healthy individuals and there was an association between reduced platelet activation and bleeding $[6,39]$. In one study, a preserved platelet aggregation response by flow cytometry identified all patients without bleeding phenotype [6]. In contrast, others found higher levels of activated fibrinogen receptor (GPIIb/IIIa) levels and P-selectin in stimulated samples in patients with AML with bleeding than without bleeding. Lower platelet activation capacity (low P-selectin expression in stimulated samples) was correlated with bleeding scores in patients with hematological cancers of whom the majority had thrombocytopenia [79]. Thus, flow cytometry is proving to be useful for studying the relationship between platelet function and bleeding in thrombocytopenia, as well as for achieving more detailed knowledge regarding the role of platelets in disease. It may also be valuable for studying extended hemostatic properties of platelets in vascular integrity. However, the method is not standardized, and the protocols are often laborious to perform, which limits their applicability, not least in urgent situations.
The protocols are also often specific for a certain condition, meaning that the test is not universally suitable as a biomarker.

\section{Platelet content}

Finally, it is noteworthy that platelets can be isolated from whole blood by centrifugation or gel-filtration. This permits evaluation of platelet constituents in platelet lysate [58]. It has been applied for studying granule release [58] and might be used to evaluate whether other platelet constituents, such as growth factors and immune mediators, influence bleeding risk in thrombocytopenia. While studies in animals, point to the relevance of platelet constituents in bleeding, human clinical studies in thrombocytopenia are lacking. Especially, information about granule constituents could be important for understanding bleeding at sites of inflammation.

Thus, several platelet function tests exist, however, most methods are limited in thrombocytopenia as the results are influenced by the low platelet count. Flow cytometry seems particularly promising for providing novel bleeding risk markers. Potentially, other relevant biomarkers can be established through studies on platelet composition and platelet adhesion.

\section{Perspectives for future research}

Future research should focus on establishing novel biomarkers of bleeding. In this regard the evaluation with flow cytometry and determination of platelet content could be helpful for research purposes. For clinical decision making, further development is, however, needed to make tests applicable for widespread use. That is, they should be affordable, easy to perform, and interpret. For currently available markers, several tests are promising, but standardization is required in order to make results from published studies comparable.

\section{Conclusions}

Platelet function varies in patients with thrombocytopenia and might identify bleeding risk. Mechanistic insight into the cause of reduced platelet function is sparse, except for some inherited platelet disorders. For the study of platelet function in thrombocytopenia, flow cytometry is a promising tool, while evaluation of platelet constituents and platelet adhesion could be relevant. An important 
message from the current paper is that the determination of bleeding risk must be tailored to specific patient populations and cannot be applied broadly to all patients with thrombocytopenia. In this regard, for example, a reliable in vitro model for prediction of effect of platelet transfusion would be helpful.

Author contributions: The author has accepted responsibility for the entire content of this submitted manuscript and approved submission.

Research funding: None declared.

Employment or leadership: None declared.

Honorarium: None declared.

Competing interests: The funding organization(s) played no role in the study design; in the collection, analysis, and interpretation of data; in the writing of the report; or in the decision to submit the report for publication.

\section{References}

1. Golebiewska EM, Poole AW. Platelet secretion: from haemostasis to wound healing and beyond. Blood Rev 2015;29:153-62.

2. Estcourt LJ, Stanworth SJ, Murphy MF. Platelet transfusions for patients with haematological malignancies: who needs them? $\mathrm{Br}$ J Haematol 2011;154:425-40.

3. Slichter SJ, Kaufman RM, Assmann SF, McCullough J, Triulzi DJ, Strauss RG, et al. Dose of prophylactic platelet transfusions and prevention of hemorrhage. N Engl J Med 2010;362:600-13.

4. Uhl L, Assmann SF, Hamza TH, Harrison RW, Gernsheimer T, Slichter SJ. Laboratory predictors of bleeding, and effect of platelet and RBC transfusions on bleeding outcomes, in the PLADO Trial. Blood 2017;130:1247-58.

5. Heddle NM, Cook RJ, Sigouin C, Slichter SJ, Murphy M, Rebulla P, et al. A descriptive analysis of international transfusion practice and bleeding outcomes in patients with acute leukemia. Transfusion 2006;46:903-11.

6. Vinholt PJ, Frederiksen H, Hvas AM, Sprogoe U, Nielsen C. Measurement of platelet aggregation, independently of patient platelet count: a flow-cytometric approach. J Thromb Haemost 2017;15:1191-202.

7. van Bladel ER, Laarhoven AG, van der Heijden LB, Heitink-Polle KM, Porcelijn L, van der Schoot CE, et al. Functional platelet defects in children with severe chronic ITP as tested with 2 novel assays applicable for low platelet counts. Blood 2014;123:1556-63.

8. Frelinger 3rd AL, Grace RF, Gerrits AJ, Berny-Lang MA, Brown T, Carmichael SL, et al. Platelet function tests, independent of platelet count, are associated with bleeding severity in ITP. Blood 2015;126:873-9.

9. Estcourt LJ, Stanworth SJ, Harrison P, Powter G, McClure M, Murphy MF, et al. Prospective observational cohort study of the association between thromboelastometry, coagulation and platelet parameters and bleeding in patients with haematological malignancies - The ATHENA study. $\mathrm{Br}$ J Haematol 2014;166:581-91.
10. Vinholt PJ, Knudsen GH, Sperling S, Frederiksen H, Nielsen C. Platelet function tests predict bleeding in patients with acute myeloid leukemia and thrombocytopenia. Am J Hematol 2019;94:891-901.

11. Gaydos LA, Freireich EJ, Mantel N. The quantitative relation between platelet count and hemorrhage in patients with acute leukemia. N Engl J Med 1962;266:905-9.

12. Hersh EM, Bodey GP, Nies BA, Freireich EJ. Causes of death in acute leukemia: a ten-year study of 414 patients from 19541963. J Am Med Assoc 1965;193:105-9.

13. Duke WW. The relation of blood platelets to hemorrhagic disease. J Am Med Assoc 1983;250:1201-9.

14. Goel R, Ness PM, Takemoto CM, Krishnamurti L, King KE, Tobian AA. Platelet transfusions in platelet consumptive disorders are associated with arterial thrombosis and in-hospital mortality. Blood 2015;125:1470-6.

15. Estcourt LJ, Heddle N, Kaufman R, McCullough J, Murphy MF, Slichter S, et al. The challenges of measuring bleeding outcomes in clinical trials of platelet transfusions. Transfusion 2013;53:1531-43.

16. Buckley MF, James JW, Brown DE, Whyte GS, Dean MG, Chesterman $\mathrm{CN}$, et al. A novel approach to the assessment of variations in the human platelet count. Thromb Haemost 2000;83:480-4.

17. Biino G, Santimone I, Minelli C, Sorice R, Frongia B, Traglia M, et al. Age- and sex-related variations in platelet count in Italy: a proposal of reference ranges based on 40987 subjects' data. PLoS One 2013;8:e54289.

18. Hui P, Cook DJ, Lim W, Fraser GA, Arnold DM. The frequency and clinical significance of thrombocytopenia complicating critical illness: a systematic review. Chest 2011;139:271-8.

19. Neunert C, Lim W, Crowther M, Cohen A, Solberg Jr L, Crowther MA. The American Society of Hematology 2011 evidence-based practice guideline for immune thrombocytopenia. Blood 2011;117:4190-207.

20. Msaouel P, Lam AP, Gundabolu K, Chrysofakis G, Yu Y, Mantzaris I, et al. Abnormal platelet count is an independent predictor of mortality in the elderly and is influenced by ethnicity. Haematologica 2014;99:930-6.

21. Friedmann AM, Sengul H, Lehmann H, Schwartz C, Goodman S. Do basic laboratory tests or clinical observations predict bleeding in thrombocytopenic oncology patients? A reevaluation of prophylactic platelet transfusions. Transfus Med Rev 2002;16:34-45.

22. Slichter SJ. Relationship between platelet count and bleeding risk in thrombocytopenic patients. Transfus Med Rev 2004;18:153-67.

23. Wandt H, Schaefer-Eckart K, Wendelin K, Pilz B, Wilhelm M, Thalheimer $M$, et al. Therapeutic platelet transfusion versus routine prophylactic transfusion in patients with haematological malignancies: an open-label, multicentre, randomised study. Lancet 2012;380:1309-16.

24. Stanworth SJ, Estcourt LJ, Powter G, Kahan BC, Dyer C, Choo L, et al. A no-prophylaxis platelet-transfusion strategy for hematologic cancers. N Engl J Med 2013;368:1771-80.

25. de Groot PG, Urbanus RT, Roest M. Platelet interaction with the vessel wall. Handb Exp Pharmacol 2012:87-110.

26. Savage B, Saldivar E, Ruggeri ZM. Initiation of platelet adhesion by arrest onto fibrinogen or translocation on von Willebrand factor. Cell 1996;84:289-97. 
27. Savage B, Almus-Jacobs F, Ruggeri ZM. Specific synergy of multiple substrate-receptor interactions in platelet thrombus formation under flow. Cell 1998;94:657-66.

28. Jackson SP. The growing complexity of platelet aggregation. Blood 2007;109:5087-95.

29. Ho-Tin-Noe B, Demers M, Wagner DD. How platelets safeguard vascular integrity. J Thromb Haemost 2011;9(Suppl 1):56-65.

30. Goerge T, Ho-Tin-Noe B, Carbo C, Benarafa C, Remold-O'Donnell $\mathrm{E}$, Zhao BQ, et al. Inflammation induces hemorrhage in thrombocytopenia. Blood 2008;111:4958-64.

31. Deppermann C, Kraft P, Volz J, Schuhmann MK, Beck S, Wolf $\mathrm{K}$, et al. Platelet secretion is crucial to prevent bleeding in the ischemic brain but not in the inflamed skin or lung in mice. Blood 2017;129:1702-6.

32. Boulaftali Y, Hess PR, Getz TM, Cholka A, Stolla M, Mackman N, et al. Platelet ITAM signaling is critical for vascular integrity in inflammation. J Clin Invest 2013;123:908-16.

33. Matus V, Valenzuela G, Saez CG, Hidalgo P, Lagos M, Aranda E, et al. An adenine insertion in exon 6 of human GP6 generates a truncated protein associated with a bleeding disorder in four Chilean families. J Thromb Haemost 2013;11:1751-9.

34. Ho-Tin-Noe B, Goerge T, Cifuni SM, Duerschmied D, Wagner DD. Platelet granule secretion continuously prevents intratumor hemorrhage. Cancer Res 2008;68:6851-8.

35. Italiano Jr JE, Richardson JL, Patel-Hett S, Battinelli E, Zaslavsky A, Short S, et al. Angiogenesis is regulated by a novel mechanism: pro- and antiangiogenic proteins are organized into separate platelet alpha granules and differentially released. Blood 2008;111:1227-33.

36. Kapur R, Semple JW. The nonhemostatic immune functions of platelets. Semin Hematol 2016;53(Suppl 1):S2-6.

37. Gresele P, Subcommittee on Platelet Physiology of the International Society on T, Hemostasis. Diagnosis of inherited platelet function disorders: guidance from the SSC of the ISTH. J Thromb Haemost 2015;13:314-22.

38. Wijgaerts A, Wittevrongel C, Thys C, Devos T, Peerlinck K, Tijssen MR, et al. The transcription factor GATA1 regulates NBEAL2 expression through a long-distance enhancer. Haematologica 2017;102:695-706.

39. Leinoe EB, Hoffmann MH, Kjaersgaard E, Nielsen JD, Bergmann OJ, Klausen TW, et al. Prediction of haemorrhage in the early stage of acute myeloid leukaemia by flow cytometric analysis of platelet function. Br J Haematol 2005;128:526-32.

40. Leinoe EB, Hoffmann MH, Kjaersgaard E, Johnsen HE. Multiple platelet defects identified by flow cytometry at diagnosis in acute myeloid leukaemia. Br J Haematol 2004; 127:76-84.

41. Greene LA, Chen S, Seery C, Imahiyerobo AM, Bussel JB. Beyond the platelet count: immature platelet fraction and thromboelastometry correlate with bleeding in patients with immune thrombocytopenia. Br J Haematol 2014;166:592-600.

42. Gunduz E, Akay OM, Bal C, Gulbas Z. Can thrombelastography be a new tool to assess bleeding risk in patients with idiopathic thrombocytopenic purpura? Platelets 2011;22:516-20.

43. Kenet G, Lubetsky A, Shenkman B, Tamarin I, Dardik R, Rechavi $\mathrm{G}$, et al. Cone and platelet analyser (CPA): a new test for the prediction of bleeding among thrombocytopenic patients. $\mathrm{Br}$ J Haematol 1998;101:255-9.

44. Psaila B, Bussel JB, Linden MD, Babula B, Li Y, Barnard MR, et al. In vivo effects of eltrombopag on platelet function in immune thrombocytopenia: no evidence of platelet activation. Blood 2012;119:4066-72.

45. Carubbi C, Masselli E, Nouvenne A, Russo D, Galli D, Mirandola P, et al. Laboratory diagnostics of inherited platelet disorders. Clin Chem Lab Med 2014;52:1091-106.

46. Vinholt PJ, Alnor A, Nybo M, Hvas AM. Prediction of bleeding and prophylactic platelet transfusions in cancer patients with thrombocytopenia. Platelets 2016;27:547-54.

47. Tripodi A. Thrombin generation assay and its application in the clinical laboratory. Clin Chem 2016;62:699-707.

48. Alkhamis TM, Beissinger RL, Chediak JR. Artificial surface effect on red blood cells and platelets in laminar shear flow. Blood 1990;75:1568-75

49. Webert K, Cook RJ, Sigouin CS, Rebulla P, Heddle NM. The risk of bleeding in thrombocytopenic patients with acute myeloid leukemia. Haematologica 2006;91:1530-7.

50. Panzer S, Jilma P. Methods for testing platelet function for transfusion medicine. Vox Sang 2011;101:1-9.

51. Kaufman RM, Djulbegovic B, Gernsheimer T, Kleinman S, Tinmouth AT, Capocelli KE, et al. Platelet transfusion: a Clinical Practice Guideline from the AABB. Ann Internal Med 2015;162:205-13.

52. Kelly AM, Garner SF, Foukaneli T, Godec TR, Herbert N, Kahan $B C$, et al. The effect of variation in donor platelet function on transfusion outcome: a semirandomized controlled trial. Blood 2017;130:214-20.

53. Thompson CB, Jakubowski JA. The pathophysiology and clinical relevance of platelet heterogeneity. Blood 1988;72:1-8.

54. Eldor A, Avitzour M, Or R, Hanna R, Penchas S. Prediction of haemorrhagic diathesis in thrombocytopenia by mean platelet volume. Br Med J (Clin Res Ed) 1982;285:397-400.

55. Lippi G, Pavesi F, Pipitone S. Evaluation of mean platelet volume with four hematological analyzers: harmonization is still an unresolved issue. Blood Coagul Fibrinolysis 2015;26:235-7.

56. Briggs C. Quality counts: new parameters in blood cell counting. Int J Lab Hematol 2009;31:277-97.

57. McDonnell A, Bride KL, Lim D, Paessler M, Witmer CM, Lambert MP. Utility of the immature platelet fraction in pediatric immune thrombocytopenia: differentiating from bone marrow failure and predicting bleeding risk. Pediatr Blood Cancer 2018;65:e26812.

58. Mumford AD, Frelinger 3rd AL, Gachet C, Gresele P, Noris $P$, Harrison $P$, et al. A review of platelet secretion assays for the diagnosis of inherited platelet secretion disorders. Thromb Haemost 2015;114:14-25.

59. Panzer S, Rieger M, Vormittag R, Eichelberger B, Dunkler D, Pabinger I. Platelet function to estimate the bleeding risk in autoimmune thrombocytopenia. Eur J Clin Invest 2007;37: 814-9.

60. Harrison P, Mackie I, Mumford A, Briggs C, Liesner R, Winter M, et al. Guidelines for the laboratory investigation of heritable disorders of platelet function. Br J Haematol 2011;155:30-44.

61. Cattaneo M, Cerletti C, Harrison P, Hayward CP, Kenny D, Nugent $D$, et al. Recommendations for the standardization of light transmission aggregometry: A Consensus of the Working Party from the Platelet Physiology Subcommittee of SSC/ISTH. J Thromb Haemost 2013;11:1183-9.

62. Seyfert UT, Haubelt H, Vogt A, Hellstern P. Variables influencing Multiplate(TM) whole blood impedance platelet aggregometry and turbidimetric platelet aggregation in healthy individuals. Platelets 2007;18:199-206. 
63. De Cuyper IM, Meinders M, van de Vijver E, de Korte D, Porcelijn $L$, de Haas $M$, et al. A novel flow cytometry-based platelet aggregation assay. Blood 2013;121:e70-80.

64. Tynngard N, Wallstedt M, Sodergren AL, Faxalv L, Ramstrom S. Platelet adhesion changes during storage studied with a novel method using flow cytometry and protein-coated beads. Platelets 2015;26:177-85.

65. Rubak P, Nissen PH, Kristensen SD, Hvas AM. Investigation of platelet function and platelet disorders using flow cytometry. Platelets 2016;27:66-74.

66. Carcao MD, Blanchette VS, Stephens D, He L, Wakefield CD, Butchart S, et al. Assessment of thrombocytopenic disorders using the Platelet Function Analyzer (PFA-100). Br J Haematol 2002;117:961-4.

67. Hans GA, Besser MW. The place of viscoelastic testing in clinical practice. Br J Haematol 2016;173:37-48.

68. Kim SY, Gu JY, Yoo HJ, Kim JE, Jang S, Choe S, et al. Benefits of thromboelastography and thrombin generation assay for bleeding prediction in patients with thrombocytopenia or hematologic malignancies. Ann Lab Med 2017;37:484-93.

69. Thompson CB, Jakubowski JA, Quinn PG, Deykin D, Valeri CR. Platelet size and age determine platelet function independently. Blood 1984;63:1372-5.

70. Tanous O, Steinberg Shemer O, Yacobovich J, Zoldan M, Horovitz $\mathrm{Y}$, Yaniv I, et al. Evaluating platelet function disorders in children with bleeding tendency - a single center study. Platelets 2017;28:676-81.

71. Sun B, Tandon NN, Yamamoto N, Yoshitake M, Kambayashi J. Luminometric assay of platelet activation in 96-well microplate. Biotechniques 2001;31:1174-81.
72. Hanke AA, Roberg K, Monaca E, Sellmann T, Weber CF, Rahe-Meyer N, et al. Impact of platelet count on results obtained from multiple electrode platelet aggregometry (Multiplate). Eur J Med Res 2010;15:214-9.

73. Michelson AD, Barnard MR, Krueger LA, Frelinger 3rd AL, Furman MI. Evaluation of platelet function by flow cytometry. Methods 2000;21:259-70.

74. Linden MD. Platelet flow cytometry. Methods Mol Biol 2013;992:241-62.

75. Shattil SJ, Hoxie JA, Cunningham M, Brass LF. Changes in the platelet membrane glycoprotein IIb.Illa complex during platelet activation. J Biol Chem 1985;260:11107-14.

76. Vinholt PJ, Frederiksen H, Hvas AM, Sprogøe U, Nielsen C. Measurement of platelet aggregation, independent of patient platelet count: a flow-cytometric approach. J Thromb Haemost 2017;15:1191-202.

77. Psaila B, Bussel JB, Frelinger AL, Babula B, Linden MD, Li Y, et al. Differences in platelet function in patients with acute myeloid leukemia and myelodysplasia compared to equally thrombocytopenic patients with immune thrombocytopenia. J Thromb Haemost 2011;9:2302-10.

78. Frelinger 3rd AL, Grace RF, Gerrits AJ, Carmichael SL, Forde EE, Michelson AD. Platelet function in ITP, independent of platelet count, is consistent over time and is associated with both current and subsequent bleeding severity. Thromb Haemost 2018;118:143-51.

79. Batman B, van Bladel ER, van Hamersveld M, Pasker-de Jong PC, Korporaal SJ, Urbanus RT, et al. Agonist-induced platelet reactivity correlates with bleeding in haemato-oncological patients. Vox Sang 2017;112:773-9. 